\title{
Stakeholders views of medicines administration by pharmacy technicians on mental health inpatient wards
}

\author{
Joanne Woodward ${ }^{1,2}\left(\mathbb{D} \cdot\right.$ Alison MacKinnon ${ }^{2} \cdot$ Richard Neil Keers ${ }^{1,3}$
}

Received: 19 February 2019 / Accepted: 4 July 2019 / Published online: 18 July 2019

(c) The Author(s) 2019

\begin{abstract}
Background The involvement of pharmacy technicians in medicines administration has been highlighted as an opportunity to enhance medicines management support for nurses and service users. Currently, there is no published evidence around this development within psychiatry. Objective To explore the perceptions of key stakeholders toward the feasibility and acceptability of pharmacy technician-led medicines administration within a mental health inpatient setting. Setting Ten acute adult and older-adult wards across five inpatient units within one UK mental health provider. Method Stratified purposeful sampling was used to recruit participants from primary (pharmacy technician, nurse and service user) and secondary (pharmacist, doctor and senior manager) stakeholder groups. One-to-one, semi-structured interviews were audio recorded, transcribed and analysed thematically using Framework analysis. Main Outcome Measure Themes arising from perspectives of stakeholders concerning the feasibility and acceptability of pharmacy technician-led medicines administration. Results Twenty participants were recruited, including twelve primary stakeholders. Attitudes towards implementation were favourable overall. Anticipated risks included de-skilling of nurses around medicines and a potentially detrimental impact on the nurse-patient therapeutic relationship; these were contrasted by potential benefits including the release of nurse time and medicines education opportunities with staff and service users. Conclusion Technician-led medicines administration was perceived as a feasible service, potentially bringing opportunities for medicines optimisation and released nursing time to care. These findings may be a source of guidance for policymakers and researchers who wish to explore the development of such services. Further exploration of safety and effectiveness is required, particularly within mental health settings.
\end{abstract}

Keywords Medicines administration · Mental health · Pharmacy technician · Qualitative research · United Kingdom

\section{Impact on Practice}

Joanne Woodward

joanne.woodward@nwbh.nhs.uk

1 School of Health Sciences, The University of Manchester, Oxford Road, Manchester M13 9PT, UK

2 North West Boroughs Healthcare NHS Foundation Trust, Hollins Park Hospital, Hollins Lane, Warrington, Cheshire WA2 8WA, UK

3 Greater Manchester Primary Care Patient Safety Translational Research Centre (GM PSTRC), Greater Manchester Mental Health NHS Foundation Trust, Manchester, UK
- Pharmacy technician-led medicines administration may be considered as a feasible and acceptable service development for mental health inpatient wards in the United Kingdom (UK).

- It is important that organisations who wish to develop pharmacy technician medicines administration services consider potential risks, benefits, barriers and facilitators; and how these may be measured or evaluated, prior to implementation.

- A summary list of considerations in relation to implementation and outcome measures has been developed, which may benefit policymakers and senior managers that wish to implement or evaluate pharmacy technician medicines administration services. 


\section{Introduction}

Nurses spend up to $40 \%$ of their workday administering medication [1], and as many people with mental health problems also have co-morbidities such as diabetes, cardiovascular disease and substance misuse [2-4], their medication regimes can be complex. Staff stress, high workloads and busy wards with numerous interruptions are all implicated in medication administration errors on mental health wards [5-8]. Furthermore, there are additional considerations relating to medicines which may be unique to psychiatry or have a higher profile than in general hospitals; including compulsory treatment under Mental Health legislation, covert administration of medicines, rapid tranquilisation, non-adherence to prescribed medication and drug-seeking behaviour [9-12]. Whilst staff may face challenges managing stressful medicines administration rounds alongside competing demands; opportunities for service users to thoroughly discuss their medication regime and any potential side-effects or problems may be overlooked, leading to increased service user anxiety relating to medicines taking, a risk factor for non-adherence [13, 14].

In England, National Health Service (NHS) mental health services are under pressure [15], with $15 \%$ fewer mental health nurses working on inpatient wards in 2014 than in 2009 [16]. The use of section powers under the Mental Health Act (1983) [17] continues to rise [18], and many mental health Trusts are operating above maximum bed occupancy levels, leaving wards overcrowded and understaffed [19]. Patient deaths on inpatient wards have been linked with staffing issues such as shortages of experienced nursing staff and high turnover rates [15, 20].

The development and expansion of clinical pharmacy services has been high on the United Kingdom (UK) government agenda for the last two decades. Pharmacy staff are acknowledged as having an important role in optimising medicines use to reduce risk and increase cost effectiveness; [1] policy initiatives now focus on utilising their skills and integrating pharmaceutical care into frontline services [21-24]. For UK Pharmacy Technicians (PTs) in both primary (community pharmacy and General Practitioner surgeries), and secondary care (hospital and specialist service) settings, their role has evolved from that of dispenser to performing more clinical, ward-based or patient-facing tasks such as medicines reconciliation, counselling on new medicines, conducting physical health assessments (such as blood tests, urinalysis, spirometry and blood pressure readings), performing medication brand switches, advising on local prescribing guidelines and final accuracy checking of dispensed medications [25-27]. This role expansion into clinical care was assisted by the introduction of mandatory registration of British (English, Welsh and Scottish) PTs with a regulatory body, the General Pharmaceutical Council $(\mathrm{GPhC})$, in 2007. In order to register, PTs must undertake 2 years consecutive work experience and complete knowledge and competency based qualifications at level 3 on the relevant National Qualifications Framework, from an approved training provider.

As registered practitioners in Britain, there is potential for PTs to administer medicines in their own right; and the shift into patient-facing roles has highlighted the development of clinical skills which could support them in undertaking medicines administration. The same right naturally extends to pharmacists, however the focus for pharmacists has shifted towards non-medical prescribing [28] as opposed to medicines administration. Pharmacy assistants could potentially reduce nursing burden by assisting with medicines administration [29], but as unregistered and unregulated practitioners they are unlikely to have the clinical skills or necessary governance arrangements required to undertake this role alone.

There is an absence of published evidence describing PT involvement in medicines administration in psychiatry. Prior to this research, a literature review was undertaken by the researcher (JW), using a systematic search and retrieval strategy. Published studies have arisen from general hospitals across the United States (US) and UK, and are service development/case-study projects, where single organisations implemented and evaluated services based on their own requirements and priorities. Papers from the US, published during the 1970s and 80s involved a large university hospital, where PTs were employed on general wards for the sole purpose of administering medicines. Numerous papers describing and evaluating various aspects of the service were published [30-36]. However these models of working came to be considered expensive and outdated [37]. One 12 month UK study where PTs administered morning medicines on a 34 bed medical ward, noted a reduction in medication administration errors with one technician error compared to seven nurse errors. However, data were only collected from ten medicines administration rounds within the 12 month period; making it too limited to perform statistical analysis. A further study involving PT supported medicines administration in one English NHS hospital, found that nurses were supportive of the scheme. However, PTs reported they did not always directly accompany nurses on the medicines round, due to feelings that their presence was potentially intrusive $[38,39]$. Published discourse relating to PT-led medicines administration within the UK has been mainly within policy documents $[21,23,27]$ and there is an absence of evidence to guide health managers on where and how PT-led medicines administration could or should be implemented, the likely acceptability of this to existing stakeholders, and any anticipated benefits or risks. There is also no current published evidence around PT-led medicines 
administration in mental health services. For this reason, research is needed to explore the views of mental health stakeholders such as nurses and service users toward the feasibility and acceptability of PTs involvement in medicines administration. Such research could be utilised by managers and policymakers who may be interested in future implementation of these schemes.

\section{Aim of the study}

The aim of this research was to explore the perceptions of key stakeholders toward the theoretical feasibility and acceptability of introducing PT-led medicines administration within a mental health inpatient setting.

\section{Ethics approval}

This study was approved by the NHS Health Research Authority and the South Central Oxford-B Research Ethics Committee (Ref 17/SC/0442). Informed consent was obtained from all individual participants included in the study.

\section{Method}

\section{Design}

The study was a qualitative design comprising of one-toone semi-structured interviews. The research methodology utilised was that of naturalistic inquiry, considered to be suitable for pharmacy practice research due to its practical, less philosophical approach which prioritises the research aim and accurate representation of participant's views over the philosophical standpoint of the author [40].

\section{Sample and setting}

The setting was ten acute adult and older-adult wards set across five inpatient units within one NHS mental health provider in Northern England. Identified stakeholders included a primary sample of those who would be directly involved in any future delivery of PT-led medicines administration (pharmacy technicians, nurses and service users), and a secondary sample of those likely to be influential in or affected by any future service redesign (pharmacists, doctors and senior managers). Eligibility criteria for the study were: English speaking participants, aged 18 or over, having capacity to provide informed consent and permanent employment on the ward (staff) or current or recent (within 6 months) admission to the ward (service users). A stratified purposeful sampling method [41] was used to select participants recruited from each stakeholder group, to ensure adequate representation. A sample size of 20-25 participants was agreed, with final numbers being determined by the principles of data saturation [42]. Participants were recruited via poster and social media advertising and researcher attendance at ward staff and service user meetings to discuss the research.

\section{Topic guide development}

The topic guide for the interviews was developed with input from the researcher (JW), clinical mentor (AM) academic supervisor (RNK) and patient and public involvement representatives from the NHS Trust. The topic guide focussed on risks and benefits of PT-led medicines administration, barriers and facilitators to implementation, anticipated training requirements and governance arrangements.

\section{Data generation}

Data generation took place from November 2017 to March 2018. Interviews were conducted face-to-face with the participant and a trained researcher (JW), on NHS Trust premises. Written informed consent was obtained from all participants, which included the digital audio recording of interviews. These were then transcribed verbatim by the researcher (JW). The mean interview time was $29 \mathrm{~min}$ (range $=17-42 \mathrm{~min})$. All identifiable information were removed during transcription.

\section{Data analysis}

The transcribed data was coded using NVivo ${ }^{\mathrm{TM}}$ qualitative data analysis software [43], following the principles of structural coding [44]. This method was chosen as structural codes are based on elements of the interview questions; in this instance risks, benefits, barriers and facilitators. These codes informed the identification of themes and the overall perception of feasibility and acceptability. Open coding was also applied to other emerging themes; such as training, skills and governance.

The coded data was then analysed using Framework analysis [45]. Framework analysis is a common approach for interpretation of semi-structured interviews. It has been shown to be useful in multi-disciplinary healthcare research [46] and has been previously utilised in pharmacy practice research [47]. The preliminary themes and codes were utilised to form an analytical framework [48] which was shared with the academic supervisor (RNK) for discussion, as part of a cyclical process of reviewing and refining the categories and codes with the remaining data. Finally, data was charted into a framework matrix which allowed the data from each transcription to be summarised by category and theme [46] 


\section{Results}

\section{Description of sample}

Twenty stakeholders were recruited to the study from the following groups: primary stakeholders-pharmacy technician $(n=5)$, nurse $(n=4)$, service user $(n=3)$; and secondary stakeholders-pharmacist $(\mathrm{n}=3)$, senior manager $(n=4)$ and doctor $(n=1)$. To ensure participant anonymity, the information from the doctor was included within the senior manager stakeholder group. The backgrounds of the other senior managers were: nurse $(n=2)$, pharmacist $(n=1)$ and allied health professional $(n=1)$. Staff members had spent an average of 7 years in post (range $=6$ months to 20 years). Service users had spent an average of 5.5 weeks (range $=2-8$ weeks) on the inpatient ward during their most recent admission. One service user was currently admitted on a ward at the time of the interview.

\section{Summary of categories and themes}

Identified data categories and themes have been summarised in a table (Table 1).

\section{Overall feasibility and acceptability}

There was evidence of widespread acceptability of PT-led medicines administration amongst participants. Generally, the service concept was viewed as an opportunity to develop skill-mix capability and to enhance medicines-related education and support to staff and service users, as described by one senior manager: "I think that swap of knowledge, clinical knowledge, and awareness of the clinical presentation of the patients we've got with us, to me it's like, well, why on earth not?" (Senior Manager 1) Some participants voiced concerns with regards to the cost of running the service along with the capacity of PTs to deliver it: "I'm not particularly against it [PT medicines administration] but it would be on the proviso that there's backfill for the jobs they were doing." (Pharmacist 1) yet it was acknowledged that with the right support and planning, a feasibility study could be an opportunity to add to the evidence base around this role: "without that trial period then how are you going to influence the national agenda?" (Senior Manager 1)

\section{Medicines education}

The most frequently identified risk was perceived to be the de-skilling of nurses around medicines. This was particularly apparent within the senior manager group. Specifically, stakeholders reported that PT-led services may reduce exposure to medicines for nurses, which could lessen their everyday knowledge. One senior manager suggested this could potentially limit opportunities for those wishing to pursue advanced practitioner or non-medical prescribing roles: "We're trying to create non-medical prescribers which will include advanced nurse practitioners, but what you don't want to do is take away more than you have to of their experience of dealing with drugs on a day-to-day basis." (Senior Manager 5) However, the risk of de-skilling nursing staff was contrasted by the most important perceived benefit of involving PTs in medicines administration-opportunities for education and training around medicines for nurses and service users. Nurses reported that PTs generally had a more in-depth knowledge of medicines, meaning nurses could learn from working together with PTs: "I think if you look at probably, with regards to especially around contraindications and side effects, I think that a technician would probably know more, have a more advanced knowledge than a registered nurse these days" (Nurse 1)

Some stakeholders raised the possibility of group learning or peer-support sessions on the ward as a way of improving practice around medicines administration:

It would be good for them [PTs] to give formal teaching sessions maybe, to the nurses. Or peer groups with the nurses; monthly professional peer groups, because each group would learn from each other and I think each group brings a slightly different dynamic to the same thing. (Senior Manager 5)

It was highlighted by stakeholders as important to offer opportunities for service users to learn more about their medicines in preparation for discharge. Many stakeholders expressed the view that this could promote informed decision making regarding medicines, and potentially improve adherence. PT2 reflects that PTs may have more opportunities compared to nurses to facilitate medicines education with service users:

If the patient took that opportunity to ask a question, I don't know whether the nurses would be able to answer at that time and they are so busy, it's the wrong time sometimes, so I think we'd be able to answer more questions and talk about compliance because you'd have the opportunity to better educate the patients. If they chose not to take their medicines, an informed discussion could take place. (Pharmacy Technician 2)

\section{Medicines safety}

Some stakeholders believed that PTs expertise and focus on medicines would translate to safer medicines administration, primarily due to PTs being interrupted or distracted less than nurses, as well as their ability to detect prescribing errors: 
Table 1 Summary of identified categories and themes

\begin{tabular}{|c|c|c|c|c|}
\hline & Risks & Benefits & Barriers & Facilitators \\
\hline \multicolumn{5}{|l|}{ Themes } \\
\hline $\begin{array}{l}\text { Overall Feasibility and } \\
\text { Acceptability }\end{array}$ & $\begin{array}{l}\text { Ward environment/per- } \\
\text { sonal safety } \\
\text { Pharmacy capacity and PT } \\
\text { availability }\end{array}$ & $\begin{array}{l}\text { Opportunities for patient } \\
\text { medicines education and } \\
\text { optimisation } \\
\text { Increased staff skill-mix } \\
\text { Staff development oppor- } \\
\text { tunities }\end{array}$ & $\begin{array}{l}\text { Technology changes (e.g. } \\
\text { electronic prescribing } \\
\text { and medicines adminis- } \\
\text { tration system imple- } \\
\text { mentation) } \\
\text { Cultural and change } \\
\text { culture (nurses, service } \\
\text { users and pharmacy } \\
\text { staff) }\end{array}$ & $\begin{array}{l}\text { Executive-level support } \\
\text { Evidence or president } \\
\text { from other mental health } \\
\text { organisations } \\
\text { Good project management } \\
\text { and evaluation } \\
\text { Staged implementation }\end{array}$ \\
\hline Medicines Education & $\begin{array}{l}\text { De-skilling of nurses in } \\
\text { medicines use }\end{array}$ & $\begin{array}{l}\text { Service user medicines } \\
\text { education and optimisa- } \\
\text { tion opportunities } \\
\text { Opportunities for nurse } \\
\text { development (e.g. pre- } \\
\text { scribing) }\end{array}$ & & \\
\hline Medicines Safety & No difference in risk & $\begin{array}{l}\text { Safer medicines adminis- } \\
\text { tration (error and missed } \\
\text { dose reduction) }\end{array}$ & & \\
\hline $\begin{array}{l}\text { Therapeutic Relation- } \\
\text { ships }\end{array}$ & $\begin{array}{l}\text { Loss of nurse-patient } \\
\text { therapeutic relationship }\end{array}$ & $\begin{array}{l}\text { Release of nurse time for } \\
\text { quality care }\end{array}$ & & $\begin{array}{l}\text { Increased PT visibility on } \\
\text { wards }\end{array}$ \\
\hline Teamwork & $\begin{array}{l}\text { Difference in staff skill set } \\
\text { (e.g. awareness of patient } \\
\text { presentation) }\end{array}$ & $\begin{array}{l}\text { Pharmacy Technician } \\
\text { development } \\
\text { Peer support }\end{array}$ & $\begin{array}{l}\text { Lack of role understanding } \\
\text { or overlapping roles }\end{array}$ & $\begin{array}{l}\text { Promotion of team working } \\
\text { Staff involvement and } \\
\text { engagement } \\
\text { Clarity around role defini- } \\
\text { tion for nurses and PTs }\end{array}$ \\
\hline Skills and Training & & $\begin{array}{l}\text { Clinical skills training for } \\
\text { PTs } \\
\text { 'Soft-skills' training for } \\
\text { PTs } \\
\text { PTs being currently } \\
\text { competent, no training } \\
\text { needed }\end{array}$ & $\begin{array}{l}\text { Queries over requirement } \\
\text { for accuracy checking } \\
\text { Pharmacy culture, expan- } \\
\text { sion to patient-facing } \\
\text { roles }\end{array}$ & $\begin{array}{l}\text { Formal competency assess- } \\
\text { ment and/or qualifications } \\
\text { Standardisation of training } \\
\text { for PTs and nurses (same } \\
\text { training) } \\
\text { Good supervision arrange- } \\
\text { ments }\end{array}$ \\
\hline Governance & & & $\begin{array}{l}\text { Lack of current evidence } \\
\text { base for mental health }\end{array}$ & $\begin{array}{l}\text { Trust policies and Standard } \\
\text { Operating Procedures } \\
\text { National guidance or policy } \\
\text { Position statements from } \\
\text { relevant } \\
\text { professional bodies } \\
\text { Risk assessment and scop- } \\
\text { ing exercises }\end{array}$ \\
\hline
\end{tabular}

They [pharmacy technicians] are devoted purely and utterly to doing that particular element of the job. So they're less likely to be interrupted, or have a patient or another nurse or doctor come in and try to accost them because they're a pharmacy technician. (Nurse 4)

However, the fact that it was mentioned less than medicines education, suggests that participants may not consider differences in medicines safety to be the primary reason for implementation. Furthermore, PTs and pharmacists were more cautious and stated that any medication safety risks would potentially remain the same due to the influence of human factors:
I think the risks would still be the same, [as nurses] you could still give the wrong thing, couldn't you cos it's still human approach isn't it? So, I don't know whether there would be any more or less, I think the risks would roughly be the same. (Pharmacy Technician 4)

\section{Therapeutic relationships}

Some stakeholders highlighted PT-led medicines administration as potentially having a negative impact on the therapeutic relationship between the nurse and service user. As one service user participant described, the medicines round was viewed by some nurses as an opportunity to assess a 
service users' presentation on a one-to-one basis, which may be lost if medicines were administered by the PT: “... because they [nurses] have quite a lot of other pressures, paperwork and stuff like that, sometimes it's difficult for them to gain contact time with patients and I think that's an ideal opportunity, if you like, an ongoing assessment" (Service User 2). However, nurses admitted to feeling underpressure to complete medicines administration within the required timescale, as a result of both nurses and service users attempting to utilise medicines administration as an opportunity for one-to-one engagement, particularly when discussions involved issues that were not medicines related. "It's very difficult to get your medicines done within the two hour time limit.... You've got to concentrate with no interruptions to get it done." (Nurse 4) The benefit of using medicines administration as a time for therapeutic interactions was viewed by other stakeholders as being minimal: "I don't think it's an interaction that's particularly therapeutic in that way, it is a task, so I'm not sure that there'd be anything lost through that [having PT-led administration]." (Senior Manager 3)

Most stakeholders reported that relationships between nurses and service users could be improved by relocating non-medicines related patient interactions within the medicines round to other times when nurses could focus their attention exclusively on such matters. This could be facilitated by releasing nurse time though greater PT involvement in medicines administration, refocussing the medicines round back to medicines related discussions. These other activities included administrative duties and therapeutic interventions with service users such as recovery focussed planning, activities, talking therapies and discussions around other social support:

We all know how thin nurses' time is spread, between documentation, speaking to the patients and doing medication rounds and ward reviews. I think if we were to help out in some way with medicines administration, that takes some responsibility off them where they can better document things that have been discussed in ward rounds, get more involved with patients and spend more time with them, where their time should be spent, in my opinion. (Pharmacy Technician 1)

It was acknowledged that PTs could benefit from opportunities to learn from nurses regarding the clinical presentation of service users, and that involving PTs in medicines administration may enhance the therapeutic relationship between service users and pharmacy technicians:

I think it may aid them in feeling more included as part of a team. And actually that direct involvement I think it'd enhance their, potentially enhance, the therapeutic relationship with our service users. At the moment of course they have contact with our patients, but it's generally more specific to serious medications the likes of lithium, clozapine, where you know some education is needed about concordance, what the processes are about monitoring. (Nurse 3)

\section{Teamwork}

Some stakeholders suggested an alternative model of collaborative working, where the PT and nurse worked together on medicines administration, created an opportunity to make the most of their complementary skills. Participants across all stakeholder groups suggested that this collaborative approach may give nurses more time to undertake clinical aspects of administration whilst the technician performed the technical preparation of medicines, and would potentially alleviate concerns around de-skilling of nurses, particularly in the early stages of implementation:

You're using the best skill-sets for both staff members and also it's not necessarily de-skilling the nurses. If anything it's skilling the nurses up more, because they'll learn a bit more about the medicines and vice versa with the technician, they learn a bit more about the physical aspects and the assessment (Pharmacist 1)

\section{Discussion}

This is the first study to prospectively explore the feasibility and acceptability of PT-led medicines administration in mental health; its findings supporting current UK policy direction concerning the transformation of clinical pharmacy services. A PT-led medicines administration service was broadly welcomed by participants, with the majority reporting a positive potential impact. Whilst participants reported potential limitations such as the de-skilling of nurses around medicines and a potential detrimental effect on the nursepatient therapeutic relationship, these were counterbalanced by contrasting accounts of benefits in terms of medicines education opportunities for both staff and service users and a release of nurse time to provide therapeutic activities and recovery planning.

The primary benefit of PT-led medicines administration was reported by participants as opportunities for service user education around medicines, the enhancement of medicines optimisation and shared decision-making. This differs from the primary focus of many acute trust schemes-medicines safety [39, 49-51], and may be due to differences in patient population within mental health services, where medicines adherence is a global public health concern [11, 52]. A lack of knowledge regarding medicines usage and potential side-effects among service users are known risk factors 
for non-adherence [53] which can cause relapse of illness, resulting in poor quality of life, reduced efficacy of further treatment, waste of healthcare resources and increased risk of suicide [52].Given this, increased medicines related knowledge and adherence should be considered as a primary outcome measure for any future implementation within mental health.

This study highlights the importance of measuring the impact of PT-led services on nurses' knowledge and skills. Nurses in this study agreed that working more closely with PTs could possibly increase their medicines-related knowledge, which may help with development into advanced practitioner roles. Most stakeholders agreed that if PT-led medicines administration was implemented, nurses would need to retain involvement in some capacity. This view is supported by published evidence from US hospitals, where PT medicines administration services ended due to the perceived safety implications of nurses becoming de-skilled in medicines administration [35, 37]. Whilst PT roles in UK general hospitals are currently being extended into 7 day working [22], this does not include $24 \mathrm{~h}$ ward cover and is not the case for many mental health Trusts, where PTs usually provide ward cover during office hours. Therefore, it is probable that models where some nurse involvement is retained; either by alternating medicines rounds with PTs or by PTs working with nurses to administer, may be preferred over models where PTs took overall responsibility.

The release of nurse time is also highlighted in a recent landmark report by Lord Carter: 'Making better use of pharmacy staff to review medicines choice and optimise supply and administration of medicines enables trusts to release significant amounts of nursing time back to clinical care' [23 p67]. However, the degree to which nurse time is released depends on the level of involvement they retain-whilst joint working with a PT may release some time during the medicines round; this would be less than a PT completing a medicines round without nurse involvement. Future research observing such changes could be conducted by way of ethnographic observations of medicines related activities [54].

The results of this research add significantly to current discourse concerning PT-led medicines administration, particularly within mental health. This research could potentially be used by British mental health organisations to fulfil the recommendations of Lord Carter's report [23], that NHS Trusts identify opportunities for innovative use of pharmacy staff to support medicines administration. Further research is needed which builds upon these findings - to design pilot or feasibility studies. As such, a summary list of considerations has been produced based on the collective accounts of participants (Text box 1). These considerations, alongside any future research, could be used as a baseline guide for service planning and implementation by others who may wish to further explore the role of PTs in medicines administration.
This may be timely, as many organisations seek to innovate in how they deliver services.

Text Box 1 Summary list of considerations

Carefully consider the service model and level of nurse involvement, in relation to the impact on de/up-skilling of nurses, released nurse time, clinical assessment of service users and cost-effectiveness of the service

Identify the resource required for both feasibility work and potential larger scale roll-out

Consider compatibility of PT working hours with availability to administer medicines without impacting on other duties, such as medicines reconciliation

Be mindful of cultural issues, which may be overcome by engagement and involvement of stakeholders affected by organisational changes

Develop a robust project plan with clearly defined roles and outcome measures, with involvement from stakeholders, overseen by a multidisciplinary project group

Develop a communications and engagement plan for stakeholder consultation or feedback

Involve senior leaders from the organisation to champion the project

Ensure governance and indemnity issues are in place to protect staff and service users

Implement a robust training pathway for PTs, with possible alignment to the nurse training pathway

Consider PT time spent on the ward, to develop service user interactions and soft-skills

Consider a phased implementation, starting with regular oral medicines only

Consideration should be given to including the following as potential outcome measures:

Evaluation of changes to nurse confidence/competency in medicines administration

Evaluation of release of nurse time, including impact of any therapeutic benefits

Non-inferiority evaluation regarding medicines safety (error rates, missed doses etc.)

Evaluation of medicines knowledge, satisfaction and/or adherence for service users

Evaluation of the accuracy of clinical assessment of presentation of service users by PTs

Evaluation of cost-effectiveness; including training costs, recruitment costs, impact of dual working (where relevant) and impact on existing PT workload

The primary strength of this study is that it is the first to qualitatively explore the feasibility and acceptability of PTled medicines administration; therefore it has value to any organisation who wishes to implement such a scheme. An additional strength is the specific focus on psychiatry, with findings that highlight issues unique to this sector. Given the 
timeliness of the study in relation to recent UK guidelines, the study has potential value to healthcare leaders and policymakers. Other strengths include involvement of a variety of stakeholders, including service users, to capture the full range of views; the latter as both study participants and in the design of the study protocol.

A limitation of the study is that the stakeholders had no practical experience of PT-led medicines administration, and so factors discussed were perceived (rather than actual). Other limitations included the small sample size and single-organisation design, which limits transferability of the findings. Furthermore, the study involved only adult acute mental health wards, and so cannot be considered transferrable to other settings such as forensic, learning disability or children's mental health. The results of this research may be of interest to countries other than Great Britain; however caution should be exercised due to international differences in PT training and registration requirements and the impact of local legislation regarding medicines administration.

\section{Conclusion}

This research shows overall acceptability for PT medicines administration on mental health wards. Whilst participants broadly welcomed PT medicines administration as a service model, they raised questions regarding whether PTs should take responsibility for all medicines administration or whether a hybrid system involving both nurses and PTs may be preferable in order to build team-working and facilitate the sharing of knowledge and skills. The findings of this research identify new lines of inquiry for research in this area, and may form a foundation of guidance for health care leaders and researchers who wish to further explore and develop PT-led medicines administration in future.

Acknowledgements The authors would like to thank the members of the Patient and Public Involvement scheme from the participating organisation, for their assistance in developing the protocol and associated documents; Steven Buckley and the Council members of the College of Mental Health Pharmacy for providing peer-review; and the participants who offered time to be interviewed for the study.

Funding The researcher (JW) was funded by a National Institute for Health Research and Health Education England Integrated Clinical Academic (ICA) award. This article presents independent research funded by the National Institute for Health Research (NIHR) and Health Education England. The views expressed are those of the author(s) and not necessarily those of the National Health Service (NHS), the NIHR or the Department of Health.

Conflicts of interest The authors declare that they have no competing interests.

Open Access This article is distributed under the terms of the Creative Commons Attribution 4.0 International License (http://creativeco mmons.org/licenses/by/4.0/), which permits unrestricted use, distribution, and reproduction in any medium, provided you give appropriate credit to the original author(s) and the source, provide a link to the Creative Commons license, and indicate if changes were made.

\section{References}

1. The Audit Commission. A Spoonful of Sugar: Medicines Management in NHS Hospitals. London; 2001.

2. Brown S, Inskip H, Barraclough B. Causes of the excess mortality of schizophrenia. Br J Psychiatry. 2000;177:212-7.

3. El-Mallakh P, McPeak D, Khara M, Okoli CT. Smoking behaviors and medical co-morbidities in patients with mental illnesses. Arch Psychiatric Nurs. 2016;30(6):740-6.

4. Henderson DC, Vincenzi B, Andrea NV, Ulloa M, Copeland PM. Pathophysiological mechanisms of increased cardiometabolic risk in people with schizophrenia and other severe mental illnesses. Lancet Psychiatry. 2015;2(5):452-64.

5. Cottney A, Innes J. Medication-administration errors in an urban mental health hospital: a direct observation study. Int J Ment Health Nurs. 2015;24(1):65-74.

6. Keers RN, Williams SD, Cooke J, Ashcroft DM. Causes of medication administration errors in hospitals: a systematic review of quantitative and qualitative evidence. Drug Saf. 2013;36(11):1045-67.

7. Hemingway S, McCann T, Baxter H, Smith G, Burgess-Dawson $\mathrm{R}$, Dewhirst K. The perceptions of nurses towards barriers to the safe administration of medicines in mental health settings. Int $\mathbf{J}$ Nurs Pract. 2017;21(6):733-40.

8. Keers RN, Placido M, Bennett K, Clayton K, Brown P, Ashcroft DM. Understanding the causes of medication administration errors in a mental health hospital using qualitative interviews with the critical incident technique. Pharmacoepidemiol Drug Saf. 2016;25:14-5.

9. Haw C, Stubbs J. Administration of medicines in food and drink: a study of older inpatients with severe mental illness. Int Psychogeriatr. 2010;22(3):409-16.

10. Calver L, Drinkwater V, Isbister GK. A prospective study of high dose sedation for rapid tranquilisation of acute behavioural disturbance in an acute mental health unit. BMC PSYCHIATRY. 2013;13:225.

11. Cramer JA, Rosenheck R. Compliance with medication regimens for mental and physical disorders. Psychiatr Serv. 1998;49(2):196-201.

12. Clark RE, Xie H, Brunette MF. Benzodiazepine prescription practices and substance abuse in persons with severe mental illness. J Clin Psychiatry. 2004;65(2):151.

13. Duxbury JA, Wright KM, Hart A, Bradley D, Roach P, Harris N, et al. A structured observation of the interaction between nurses and patients during the administration of medication in an acute mental health unit. J Clin Nurs. 2010;19(17-18):2481-92.

14. Duxbury JA, Wright KM, Bradley DM, Roach P. A study of the views of patients and nurses about the administration of medication in the acute mental health setting. J Psychiatric Mental Health Nurs. 2009;16(7):672-7.

15. Gilburt H. Mental health under pressure. London: The Kings Fund; 2016.

16. Addicott R, Maguire D, Honeyman M, Jabbal J. Workforce planning in the NHS. London; 2015.

17. Department of Health. The Mental Health Act. 1983 [cited 10/08/2018]; https://www.legislation.gov.uk/ukpga/1983/20/conte nts.

18. NHS Digital. Inpatients formally detained in hospitals under the Mental Health Act 1983 and patients subject to Supervised 
Community Treatment: 2015/16, Annual figures. NHS Digital; 2018.

19. Crisp N, Smith G, Nicholson K. Old problems, new solutionsimproving acute psychiatric care for adults in England. The Commision on Acute Adult Psychiatric Care. 2016. p. 25.

20. National Confidential Inquiry into Suicide and Homicide by People with Mental Illness (NCISH). Healthy services and safer patients: linke between patient suicide and features of mental health care providers. Manchester; 2015.

21. Lord Carter of Coles. Operational productivity and performance in English NHS acute hospitals: unwarranted variations. London: Department of Health; 2016.

22. Office of the Chief Pharmaceutical Officer. Transformation of seven day clinical pharmacy services in acute hospitals. London: NHS England; 2016.

23. Lord Carter of Coles. NHS operational productivity: unwarranted variances in mental health services and community health services. NHS Improvement: Department of Health; 2018.

24. The Royal Pharmaceutical Society. No Health without Mental Health: How can pharmacy support people with mental health problems? London; 2018.

25. Thomas M, Cross S, Nagra M, Bullock A. Empowering technicians to work in extended clinical roles. Pharm J R Pharm Soc G B. 2008; 15:145.

26. Boughen M, Fenn T, Croot J, Frost K, Family H, Wright D, et al. Identifying the Roles of Pharmacy Technicians in the UK: University of East Anglia \& Association of Pharmacy Technicians UK; 2016

27. Boughen M, Sutton J, Fenn T, Wright D. Defining the role of the pharmacy technician and identifying their future role in medicines optimisation. Pharm J Pharm Edu Pract. 2017;5(3):40.

28. Mulholland PJ. Pharmacists as non-medical prescribers: what role can they play? The experience in a neonatal intensive care unit. Eur J Hospital Pharm. 2014;21(6):335-8.

29. Baqir W, Jones K, Horsley W, Barrett S, Fisher D, Copeland R, et al. Reducing unacceptable missed doses: pharmacy assistant-supported medicine administration. Int J Pharm Pract. 2015;23(5):327-32.

30. Shoup LK. A pharmacy coordinated unit dose dispensing and drug administration system. Recruitment, selection and training of pharmacy technicians. Am J Hosp Pharm. 1970;27(11):907-10.

31. Lachner BJ. A pharmacy coordinated unit dose dispensing and drug administration system. Administrative implications. Am J Hosp Pharm. 1970;27(11):899-901.

32. Berry CC. A pharmacy coordinated unit dose dispensing and drug administration system. Description of the system. Am J Hosp Pharm. 1970;27(11):890-8.

33. Martin RM. A pharmacy coordinated unit dose dispensing and drug administration system. Nursing implications. Am J Hosp Pharm. 1970;27(11):902-6.

34. Cortese LM, Greenberger DW, Schneider PJ, Bourret JA. Job characteristics and satisfaction of pharmacy technicians. Am J Hosp Pharm. 1987;44(11):2514-8.

35. Fillmore AD, Schneider PJ, Bourret JA, Caswell RJ. Costs of training drug-administration technicians. Am J Hosp Pharm. 1986;43(7):1706-9.

36. Scala SM, Schneider PJ, Smith GL, Bourret JA. Activity analysis of pharmacy-directed drug-administration technicians. Am J Hosp Pharm. 1986;43(7):1702-6.

37. Thompson CA. When pharmacy managed drug administration. Am J Health-Syst Pharm. 2008;65(19):1793-4.

38. Seston EM, Ashcroft DM, Lamerton E, Harper L, Keers RN Evaluating the implementation and impact of a pharmacy technician-supported medicines administration service designed to reduce omitted doses in hospitals: a qualitative study. BMC Health Serv Res. 2019;19(1):325.

39. Keers RN, Seston E, Kontopantelis E, Lamerton E, Burgess A, Morris F, et al. Evaluation of pharmacy TECHnician supported MEDication administration rounds (TECHMED) on reducing omitted doses: a pilot randomised controlled trial and process evaluation in a university teaching hospital: Centre for Pharmacoepidemiology and Drug Safety, Division of Pharmacy and Optometry, School of Health Sciences, The University of Manchester, Manchester Academic Health Sciences Centre (MAHSC); 2017.

40. Auta A, Strickland-Hodge B, Maz J. There is still a case for a generic qualitative approach in some pharmacy practice research. Amsterdam: Elsevier Inc.; 2017. p. 266-8.

41. Creswell JW. Qualitative inquiry and research design: choosing among five approaches. Thousand Oaks: SAGE Publications; 2012.

42. Fusch PI, Lawrence R. Are we there yet? Data saturation in qualitative research. Qual Rep. 2015;20(9):1408-16.

43. QSR International Pty Ltd. NVivo Qualitative Data Analysis Software. 10 ed; 2012.

44. Saldaña J. The coding manual for qualitative researchers. Los Angeles: SAGE; 2009.

45. Ritchie J, Lewis J. Qualitative research practice: a guide for social science students and researchers. Thousand Oaks: SAGE Publications; 2003.

46. Gale NK, Heath G, Cameron E, Rashid S, Redwood S. Using the framework method for the analysis of qualitative data in multi-disciplinary health research. BMC Med Res Methodol. 2013;13(1): 117

47. Moullin JC, Sabater-Hernández D, Benrimoj SI. Qualitative study on the implementation of professional pharmacy services in Australian community pharmacies using framework analysis. London: BMC Health Services Research; 2016.

48. Gale NK, Shapiro J, McLeod HST, Redwood S, Hewison A. Patients-people-place: developing a framework for researching organizational culture during health service redesign and change. Implement Sci. 2014;9(1):106.

49. McLean D. Medicines administration rounds can be led by pharmacy technicians. Pharm Pract. 2006;16(1):19-23.

50. Slimm M. New and emerging roles for pharmacy staff. Austria: European Association of Hospital Pharmacists Congress. Vienna; 2016.

51. Thomas K, Powell J. Administration of Medicines: Getting it right every time. The Association of Pharmacy Technicians UK Annual Conference; 2017; Cardiff, Wales; 2017.

52. Semahegn A, Torpey K, Manu A, Assefa N, Tesfaye G, Ankomah A. Psychotropic medication non-adherence and associated factors among adult patients with major psychiatric disorders: a protocol for a systematic review. Syst Rev. 2018;7:10.

53. Velligan DI, Weiden PJ, Sajatovic M, Scott J, Carpenter D, Ross $\mathrm{R}$, et al. The expert consensus guideline series: adherence problems in patients with serious and persistent mental illness. J Clin Psychiatry. 2009;70(Suppl 4):1-46.

54. Jennings BM, Sandelowski M, Mark B. The Nurse's medication day. Qual Health Res. 2011;21(10):1441-51.

Publisher's Note Springer Nature remains neutral with regard to jurisdictional claims in published maps and institutional affiliations. 